\title{
Identification of biomarkers and their functions in dasatinib-resistant pancreatic cancer using bioinformatics analysis
}

\author{
JINGSUN WEI $^{1 *}$, RONGBO HAN $^{1 *}$, XINYU SU $^{1}$, YUETONG CHEN $^{1}$, JUNFENG SHI $^{1}$, XIAOWEN CUI $^{1}$, \\ HONGHONG ZHANG ${ }^{1}$, YANG GONG ${ }^{1}, \mathrm{XIA} \mathrm{CHU}^{2}$ and JINFEI CHEN ${ }^{1,3}$ \\ ${ }^{1}$ Department of Oncology, Nanjing First Hospital, Nanjing Medical University; \\ ${ }^{2}$ Department of Oncology, Nanjing First Hospital, Southeast University, Nanjing, Jiangsu 210006; \\ ${ }^{3}$ Jiangsu Key Laboratory of Cancer Biomarkers, Prevention and Treatment, Collaborative Innovation Center for \\ Cancer Personalized Medicine, Nanjing Medical University, Nanjing, Jiangsu 211166, P.R. China
}

Received March 4, 2018; Accepted April 2, 2019

DOI: $10.3892 / 01.2019 .10281$

\begin{abstract}
Dasatinib is a tyrosine kinase inhibitor, which inhibits tumor proliferation by blocking SRC pathways and is considered as a potential treatment of various epithelial neoplasms, including pancreatic cancer. However, dasatinib efficacy is largely limited due to drug resistance. In the present study, bioinformatics strategies were used to investigate the potential mechanisms of dasatinib-resistance in pancreatic cancer. The gene expression profiles of the Panc0403, Panc0504, Panc1005 (dasatinib-sensitive), SU8686, MiaPaCa2 and Panc1 (acquired dasatinib-resistant) cell lines were obtained from the gene expression omnibus database. The differentially expressed genes (DEGs) were then selected using R software. In addition, gene ontology (GO) and pathway enrichment analysis were performed through the Database for Annotation, Visualization and Integrated Discovery. A protein-protein interaction (PPI) network was constructed and analyzed to determine the hub genes using the Search Tool for the Retrieval of Interacting Genes database. A total of 472 DEGs, including vimentin, transmembrane 41 six family member 18 and S100 calcium binding protein $\mathrm{P}$, were identified. Enrichment analysis by GO function demonstrated that DEGs were associated with extracellular components, signal regulation and binding factors. The analysis of the Kyoto Encyclopedia of Genes and Genomes demonstrated that several adenocarcinoma pathways were enriched, including the phosphoinositide 3-kinases/protein kinase B and mitogen-activated
\end{abstract}

Correspondence to: Dr Jinfei Chen, Department of Oncology, Nanjing First Hospital, Nanjing Medical University, 68 Changle Road, Nanjing 210006, P.R. China

E-mail: jinfeichen@sohu.com

*Contributed equally

Key words: dasatinib resistance, dysfunctional pathway, differentially expressed genes, functional enrichment analysis, overall survival, pancreatic carcinoma protein kinase signaling pathways. Some hub genes were highlighted following the PPI network construction, including Rac family small GTPase 1, laminin subunit $\alpha 3$, integrin subunit $\beta 4$, integrin subunit $\alpha 2$, collagen type VI $\alpha 1$ chain, collagen type I $\alpha 2$ chain, arrestin $\beta 1$ and synaptotagmin 1 , which may be associated with pancreatic adenocarcinoma prognosis. A total of five out of eight hub genes were highly associated with the overall survival rate $(\mathrm{P}<0.05)$. In conclusion, the present study reported novel insights into the mechanisms of dasatinib resistance. Identification of these hub genes may be considered as potential novel treatment targets for dasatinib-resistance in pancreatic cancer.

\section{Introduction}

Pancreatic cancer is one of the most common types of cancer worldwide and the fourth leading cause of cancer-associated mortality in developed countries $(1,2)$. Pancreatic cancer is a malignant disease with a median survival time of 3-6 months and a 5-year survival rate of less than 5\% $(3,4)$. Chemotherapy is the primary adjuvant therapy for pancreatic cancer (5). Dasatinib is an oral inhibitor of dual Bcr/Abl and Src family kinases (6) commonly used in hematopoietic tumors (7). Src kinase was one of the earliest discovered proto-oncoproteins in humans, which exhibits high activity in a number of human tumors and is involved in the process of malignant transformation of cells (8). The Src-mediated tumor cell signal transduction network serves a crucial role in tumorigenic processes, such as cell growth (9). Activated Src kinase promotes cell proliferation through the Ras/Raf/mitogen-activated protein kinase (MAPK) pathway (10). Dasatinib is also an Src inhibitor approved by the Food and Drug Administration (FDA) for the treatment of pancreatic cancer (11). A previous study reported that Dasatinib could treat epithelial neoplasms, including pancreatic cancer (12). In addition, dasatinib can slow down cancer metastasis and the progression of human pancreatic ductal adenocarcinoma (PDAC) in orthotopic mouse models, and may be able to stimulate PDAC cell apoptosis in humans and mice (13-15). Bartscht et al (16) reported that dasatinib inhibits the function of Src kinases and transforming growth factor $\beta 1$ (TGF- $\beta 1$ ) in clinical and experimental therapeutics 
to prevent the metastatic spread of late-stage PDAC. Dasatinib is a highly promising treatment of pancreatic cancer; however, most patients who have a good response to inhibitors typically experience disease recurrence due to drug-resistance development, which becomes a severe clinical problem (11).

The mechanism of acquired dasatinib resistance is unclear. Previous studies reported that SRC/TGF- $\beta$ alteration and multiple signals, such as the MAPK signaling pathways, may be associated with the progression of drug resistance $(17,18)$. Beauchamp et al (19) revealed that acquired dasatinib resistance may be related to a discoidin domain receptor tyrosine kinase 2 gatekeeper mutation and the loss of neurofibromatosis type 1 . These studies demonstrated that multiple genes participate in the development of dasatinib-resistance, and that alterations in multiple genes are often associated with cells resistance to dasatinib. Therefore, it is not advisable to study the mechanism of drug resistance through single gene changes or pathways.

Since the precise molecular mechanisms underlying dasatinib resistance remain unknown, studies on novel treatments are still in the early stages and their outcomes are not optimal; the majority of studies focus on specific molecular targets or genes, ignoring the possibility that dasatinib resistance may be due to the abnormal expression of multiple genes (20). Traditional treatment methods, which only consider one gene, may be unable to combat drug resistance (21). It is therefore crucial to investigate the resistance-associated gene variations using novel methods, including genome-wide technologies, which may provide new understanding of dasatinib resistance and allow the development of novel treatment strategy.

Microarray is a tool for high-throughput screening, which is used for the analysis of global gene expression profiles, particularly for the study of the underlying mechanisms of various diseases. In the present study, the gene expression profiles of dasatinib-resistant pancreatic cancer cells were analyzed using public microarray data to better understand the underlying mechanisms of dasatinib resistance. Bioinformatics methods were used to search for differentially expressed genes (DEGs) between dasatinib-sensitive and dasatinib-resistant pancreatic cancer cells. The functions of the DEGs were evaluated using gene ontology (GO) annotation, pathway enrichment and the construction of a protein-protein interaction (PPI) network. The present study aimed to understand the mechanisms of drug resistance and to determine potential tumor therapy targets to prevent dasatinib resistance.

\section{Materials and methods}

DEG identification from public microarray data. To identify DEGs from acquired dasatinib-resistant pancreatic cancer cells, the shared gene expression profile (GSE59357) was obtained from the Gene Expression Omnibus database (https://www.ncbi.nlm.nih.gov/geo). This dataset was uploaded by Chien et al (11). The information included the Panc0403, Panc0504, Panc1005 (dasatinib-sensitive), SU8686, MiaPaCa2 and Panc1 (dasatinib-resistant) cell lines. The dataset was analyzed using $\mathrm{R}$ software ( $\mathrm{R}$ version 3.4 .1 ; https://mirrors. tuna.tsinghua.edu.cn/CRAN). Student's t-test was utilized to screen the dasatinib resistance-associated DEGs among the cell lines, using a threshold of $\mathrm{P}<0.05$ and a fold change $\geq 1.5$.

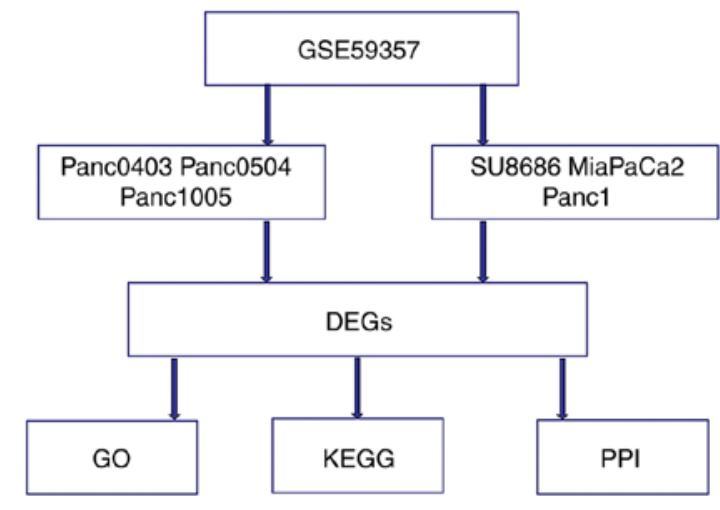

Figure 1. Flow diagram of bioinformatics analysis. DEGs, differentially expressed genes; GO, gene ontology; KEGG, Kyoto Encyclopedia of Genes and Genomes; PPI, protein-protein interaction.

Functional enrichment analysis of DEGs. The Database for Annotation, Visualization and Integrated Discovery (DAVID, https://david.ncifcrf.gov) was used to perform the functional enrichment analysis of the DEGs, including gene ontology (GO) functional analysis and Kyoto Encyclopedia of Genes and Genomes (KEGG) pathway analysis. In the GO analysis, the categories included cellular component (CC), biological process (BP) and molecular function (MF) terms, and a P-value of $<0.01$ was considered to indicate a statistically significant difference. In the KEGG pathway analysis, enriched pathways were identified based on their hypergeometric distribution with a P-value of $<0.05$.

Construction of a PPI network by Search Tool for the Retrieval of Interacting Genes (STRING). The 472 identified DEGs were analyzed by inputting the identification numbers of the DEGs into the online tool STRING (https://string-db. org). A combined score of $\geq 0.7$ (high confidence score) was considered significant. The hub proteins were chosen based on their associations with other proteins: Proteins coded by DEGs that were associated with a higher number of other DEGs were considered to serve important roles in the PPI network.

Analysis of the hub genes and their association with pancreatic adenocarcinoma prognosis. The Oncolnc website (http://www.oncolnc.org), which uses The Cancer Genome Atlas database for survival analysis of cancer patients, was used to analyze the hub genes and their association with the prognosis of patients with pancreatic cancer.

\section{Results}

Identification of the DEGs between dasatinib-sensitive and acquired dasatinib-resistant pancreatic cancer cells. The 't test' option in R software was used to research the gene expression profiles from the GSE59357 public microarray dataset. It highlighted the DEGs between Panc0403, Panc0504, Panc1005 (Dasatinib-sensitive), SU8686, MiaPaCa2 and Panc1 (acquired Dasatinib-resistant) pancreatic cancer cells. (Fig. 1). A total of 472 DEGs were identified, which comprised 333 downregulated and 139 upregulated DEGs. The top 10 upregulated and downregulated DEGs are listed in Table I. 
Table I. The top 10 downregulated and upregulated differentially expressed genes in dasatinib-resistant cell lines compared with dasatinib-sensitive cell lines.

\begin{tabular}{llll}
\hline A, Upregulated genes & & & \\
\hline Probe set & \multicolumn{1}{c}{ Gene } & $\operatorname{logFC}$ & P-value \\
\hline ILMN_2058251 & VIM & 5.050515 & $6.35 \times 10^{-5}$ \\
ILMN_2169966 & TM4SF18 & 3.8899119 & $2.27 \times 10^{-4}$ \\
ILMN_2169152 & SRGN & 3.7095162 & $1.77 \times 10^{-3}$ \\
ILMN_1804735 & $C B S$ & 3.6128934 & $1.23 \times 10^{-4}$ \\
ILMN_1757604 & $T P M 2$ & 3.5489746 & $1.07 \times 10^{-5}$ \\
ILMN_1680874 & $T U B B 2 B$ & 3.5267246 & $7.53 \times 10^{-4}$ \\
ILMN_1811468 & $I R X 3$ & 3.3427754 & $3.34 \times 10^{-5}$ \\
ILMN_1729117 & $C O L 5 A 2$ & 3.3276338 & $2.21 \times 10^{-3}$ \\
ILMN_1703178 & $S C G 2$ & 3.2029857 & $3.70 \times 10^{-3}$ \\
ILMN_1790338 & $P R R X 2$ & 3.1810426 & $1.29 \times 10^{-5}$ \\
\hline
\end{tabular}

B, Downregulated genes

\begin{tabular}{llll}
\hline Probe set & \multicolumn{1}{c}{ Gene } & $\operatorname{logFC}$ & P-value \\
\hline ILMN_1712522 & CEACAM6 & -4.1498441 & $2.22 \times 10^{-3}$ \\
ILMN_2188862 & GDF15 & -4.202082 & $6.03 \times 10^{-4}$ \\
ILMN_1666222 & PHACTR3 & -4.2059744 & $5.99 \times 10^{-5}$ \\
ILMN_2133205 & GPX2 & -4.2320599 & $7.36 \times 10^{-4}$ \\
ILMN_2353161 & MSLN & -4.2425188 & $3.35 \times 10^{-4}$ \\
ILMN_2160210 & EPCAM & -4.2522758 & $7.48 \times 10^{-4}$ \\
ILMN_2163723 & KRT7 & -4.3465465 & $1.70 \times 10^{-4}$ \\
ILMN_1692223 & LCN2 & -4.3976553 & $1.98 \times 10^{-3}$ \\
ILMN_1739001 & TACSTD2 & -5.0377579 & $9.36 \times 10^{-4}$ \\
ILMN_1801216 & $S 100 P$ & -5.2731601 & $9.94 \times 10^{-4}$ \\
\hline
\end{tabular}

FC, fold change.

The functional annotation and pathway enrichment of $D E G s$. To investigate the biological functions of the identified DEGs, GO analysis was used in DAVID to cluster the data. The enriched GO terms were separated into CC, BP and MF ontology categories (Fig. 2).

In the CC ontology (Fig. 2A), most of the enriched categories were associated with cell membrane components, including 'extracellular region' (175 genes), 'membrane-bounded vesicles' (160 genes) and 'extracellular vesicles' (126 genes). In addition, the other highly enriched terms included 'extracellular organelles' (126 genes), 'the extracellular region' (162 genes) and 'extracellular exosomes' (125 genes). In the BP ontology (Fig. 2B), the highest number of DEGs were enriched in regulation-associated terms, including 'signal regulation' (118 genes), 'cell communication regulation' (118 genes), 'molecular function regulation' (117 genes) and 'signal transduction regulation' (107 genes). A number of enriched categories were associated with transduction, apoptosis and localization, such as 'cell proliferation' (95 genes), 'intracellular signal transduction' (100 genes), ‘apoptotic processes’ (73 genes) and 'macromolecule localization' (97 genes). In the MF ontology (Fig. 2C), regulator-associated and binding-associated components accounted for the majority of the enriched GO categories, including 'molecular function regulation' (61 genes), 'identical protein binding' (57 genes), 'enzyme regulator activity' (43 genes) and 'cytoskeletal protein binding' (35 genes).

Furthermore, the KEGG pathway analysis identified dysfunctional enriched pathways, including "pathways in cancer' (26 genes), 'phosphoinositide 3-kinase (PI3K)-protein kinase B (Akt) signaling pathway' (20 genes), 'MAPK signaling pathway' (16 genes), 'transcriptional misregulation in cancer' (13 genes) and 'p53 signaling pathway' (6 genes; Table II).

Construction of a PPI network. STRING, which is a database used for the prediction of protein association, was used to predict the protein interactions among the identified DEGs. Firstly, the 472 DEGs were entered in the STRING website to obtain their PPI data. Then, if the combined score was $\geq 0.7$, the PPIs were chosen to construct a PPI network. In the PPI 
A

$\mathrm{cc}$

GO:0005576 extracellular region GO:0044421 extracellular region part GO:0031988 membrane-bounded vesicle GO:0043230 extracellular organelle

GO:0070062 extracellular exosome GO:0005829 cytosol Go.0030054 cell junction GO:0005615 extracellular space GO:0098590 plasma membrane region GO:0016023 cytoplasmic, membrane-bounded vesicle
GO:0097458 neuron par GO:0070161 anchoring junction GO:0005912 adherens junction

GO:0043005 neuron projection
GO:0009986 cell surtace GO:0009986 $\sim$ cell surface

GO:0005911 cell-cell junction

GO:0031012 extracellular matrix GO:0099513-polymeric cytoskeletal fiber

GO:0031252 cell leading edge

GO:0015629 actin cytoskeleton G0:0030424-axon

GO:0005924 cell-substrate adherens junction

GO:0005925 tocal adhesion

GO:0045177 apical part of cell

GO:0031253 cell projection membrane

$\begin{array}{rl}\text { GO:0016324 apical plasma membrane } 22 & 22 \\ \text { GO:0005578 proteinaceous extracellular matrix } 21 & 22\end{array}$

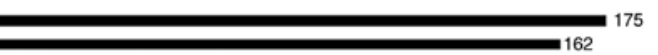

126
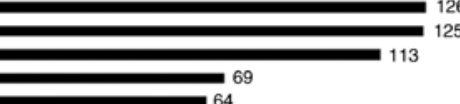

58

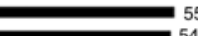

41

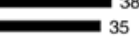

31
31

28

28

$20 \quad 40$

60

80

100

120

$160 \quad 180$

200

B

GO:0023051 regulation of signaling

GO:00650 regulation of cell communication

GO:0009966 regulation of signal transduction

GO:0010033 response to organic substance

GO:0050790 regulation of catalytic activity

GO:0009893 positive regulation of metabolic process

GO:0035556 intracellular signal transduction

GO:0007166 cell surface receptor signaling pathway GO:0008283 cell proliferation

GO:0010604 positive regulation of macromolecule metabolic.

GO:0031325 positive regulation of cellular metabolic proces
GO:0070887 cellular response to chemical stimulus

GO:0051246 regulation of protein metabolic process

GO:0032268 regulation of cellular protein metabolic process

GO:0006928 movement of cell or subcellular componen

GO:0042127 regulation of cell proliferation
GO:0008219 cell death

GO:0022610 - biological adhesion

GO:0051128 regulation of cellular component organization

GO:0009605 response to external stimulus GO:0007155 cell adhesio

GO:0008104 protein localization GO:0040011 locomotion GO:0051049 regulation of transport

GO:0031324 negative regulation of cellular metabolic process
GO:0048584 positive regulation of response to stimulus

GO:000399־nervous system development

BP

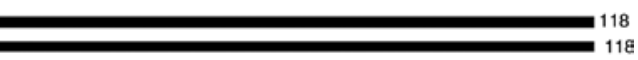

107

102
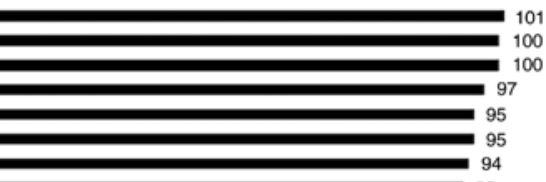

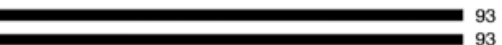
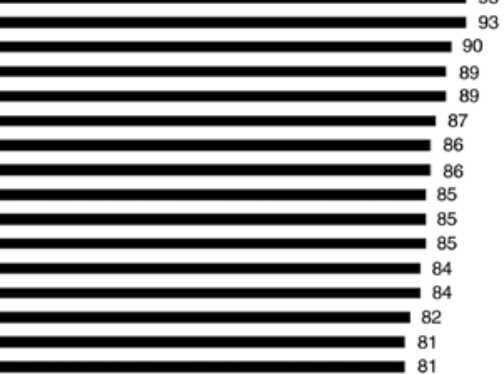

\section{0}

40

60

80

100

120

C

GO:0098772 molecular function regulator GO:0042802 identical protein binding GO:0030234 enzyme regulator activity GO:0005198 structural molecule activity GO:0008092 cytoskeletal protein binding GO:0019904 protein domain specific binding GO:0005509 calcium ion bindin

GO:0050839 cell adhesion molecule bindin GO:0019900 kinase binding

GO:0019901 protein kinase binding

GO:0004857 enzyme inhibitor activity GO:0003779 actin binding

GO:0098631 protein binding involved in cell adhesio GO:0098632 protein binding involved in cell-cell adhsion
GO:0098641 cadherin binding involved in cell-cell adhsion GO:0003924 - GTPase activity

GO:0019838 growth factor binding

GO:0019207 -kinase regulator activity

GO:0019887 protein kinase regulator activity

GO:0005200 structural constituent of cytokeleton GO:0005178 integrin bindin

GO:0001948 glycoprotein binding

GO:009210 kinase inhibitor activity

GO:0004860 protein kinase inhibitor activity GO:0005518 collagen binding

GO:003029 - protein serine/threonine kinase inhibitor activity

GO:000403 aldo-keto reductase (NADP) activit

GO:0004861 cyclin-dependent protein serine/threonine kinase inhibitor activity

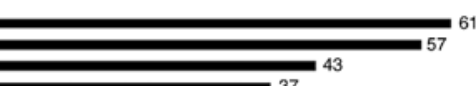

37

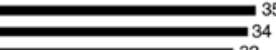

32
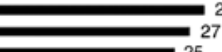

24
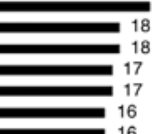

14

10

8

7

6

${ }_{0}^{4}$

Figure 2. DEGs analyzed by GO enrichment. (A) GO enrichment of DEGs in CC ontology ( $>20$ genes). (B) GO enrichment of DEGs in BP ontology (>80 genes) (C) GO enrichment of DEGs in MF ontology ( $>4$ genes). DEGs, differentially expressed genes; GO, gene ontology; CC, cellular component; BP, biological process; MF, molecular function. 
Table II. Enriched Kyoto Encyclopedia of Genes and Genomes pathway analysis of the differentially expressed genes.

\begin{tabular}{|c|c|c|}
\hline Term & Count & P-value \\
\hline hsa05200: Pathways in cancer & 26 & $1.31 \times 10^{-4}$ \\
\hline hsa04151: PI3K-Akt signaling pathway & 20 & $4.54 \times 10^{-3}$ \\
\hline hsa04010: MAPK signaling pathway & 16 & $6.31 \times 10^{-3}$ \\
\hline hsa04510: Focal adhesion & 15 & $2.28 \times 10^{-3}$ \\
\hline hsa05202: Transcriptional misregulation in cancer & 13 & $3.10 \times 10^{-3}$ \\
\hline hsa05205: Proteoglycans in cancer & 12 & $2.83 \times 10^{-2}$ \\
\hline hsa04512: ECM-receptor interaction & 11 & $1.74 \times 10^{-4}$ \\
\hline hsa04530: Tight junction & 10 & $1.66 \times 10^{-2}$ \\
\hline hsa04514: Cell adhesion molecules (CAMs) & 10 & $2.05 \times 10^{-2}$ \\
\hline hsa04670: Leukocyte transendothelial migration & 9 & $1.97 \times 10^{-2}$ \\
\hline hsa04550: Signaling pathways regulating pluripotency of stem cells & 9 & $4.74 \times 10^{-2}$ \\
\hline hsa05146: Amoebiasis & 8 & $3.20 \times 10^{-2}$ \\
\hline hsa05212: Pancreatic cancer & 7 & $1.04 \times 10^{-2}$ \\
\hline hsa04610: Complement and coagulation cascades & 7 & $1.38 \times 10^{-2}$ \\
\hline hsa05222: Small cell lung cancer & 7 & $3.46 \times 10^{-2}$ \\
\hline hsa04540: Gap junction & 7 & $4.00 \times 10^{-2}$ \\
\hline hsa05219: Bladder cancer & 6 & $5.95 \times 10^{-3}$ \\
\hline hsa05130: Pathogenic Escherichia coli infection & 6 & $1.48 \times 10^{-2}$ \\
\hline hsa04115: p53 signaling pathway & 6 & $4.24 \times 10^{-2}$ \\
\hline hsa00982: Drug metabolism-cytochrome P450 & 6 & $4.47 \times 10^{-2}$ \\
\hline hsa04978: Mineral absorption & 5 & $4.19 \times 10^{-2}$ \\
\hline
\end{tabular}

ECM, extracellular matrix; MAPK, mitogen-activated protein kinase.

Table III. The eight key hub genes and its associated genes.

\begin{tabular}{|c|c|}
\hline Hub gene & Associated genes \\
\hline$A R R B 1$ & FZD4, F2R, DTX2, SYT1, AP1M2, TPD52L1, REPS2, FNBP1 \\
\hline SYT1 & SNAP25, GAD1, ARRB1, FZD4, REPS2, FNBP1, EXPH5 \\
\hline COL1A2 & COL3A1, COL5A2, LEPRE1, COL6A1, SMAD4, ITGA2, PLOD1, ITGB4 \\
\hline COL6A1 & COL3A1, COL1A2, COL5A2, PLOD1, ITGA2, LEPRE1, ITGB4 \\
\hline ITGA2 & COL3A1, COL1A2, LAMC2, LAMA3, COL6A1, ITGB4, FLNB, LAMA5 \\
\hline ITGB4 & LAMC2, FLNB, LAMA3, LAMA5, ERBB3, SFN, PMP22, ITGA2, JUP, COL1A2, COL6A1 \\
\hline$L A M A 3$ & LAMC2, ITGB4, NTN4, ITGA2, MMP7, LAMA5, RAC1 \\
\hline$R A C 1$ & $\begin{array}{l}\text { MYH10, CYFIP2, DOCK5, JUP, MYL9, SYK, EPHA1, ARHGEF5, ARAP3, DOCK9, } \\
\text { DOCK11, ABLIM3, EFNB2, DLC1, NRP1, FGD3, LAMA3, LAMC2, LAMA5 }\end{array}$ \\
\hline
\end{tabular}

ARBB1, arrestin $\beta 1$; COL1A2, collagen type I $\alpha 2$ chain; COL6A1, collagen type VI $\alpha 1$ chain; ITGA2, integrin subunit $\alpha 2$; ITGB4, integrin subunit $\beta 4$; LAMA3, laminin subunit $\alpha 3$; RAC1, Rac family small GTPase 1; SYT1, synaptotagmin 1.

network, eight node proteins, including arrestin $\beta 1$ (ARRB1), synaptotagmin 1 (SYT1), collagen type I $\alpha 2$ chain (COL1A2), collagen type VI $\alpha 1$ chain (COL6A1), integrin subunit $\beta 4$ (ITGB4), integrin subunit $\alpha 2$ (ITGA2), laminin subunit $\alpha 3$ (LAMA3) and Rac family small GTPase 1 (RAC1) had a high association with other node proteins $(>7)$, which demonstrated their increased hub degrees (Table III; Fig. 3). The hub genes may therefore serve a crucial role in dasatinib resistance within pancreatic cancer.
Association between hub genes and pancreatic adenocarcinoma prognosis. The Oncolnc website was used to analyze the hub genes and five hub genes were found to have a strong association with pancreatic adenocarcinoma prognosis in 174 patients (Fig. 4). Kaplan-Meier survival analysis demonstrated that high expression levels of the hub genes COL1A2, ITGB4, ITGA2, LAMA3 and RAC1 were associated with a poorer overall survival in 174 patients with pancreatic adenocarcinoma compared with patients with low expression 


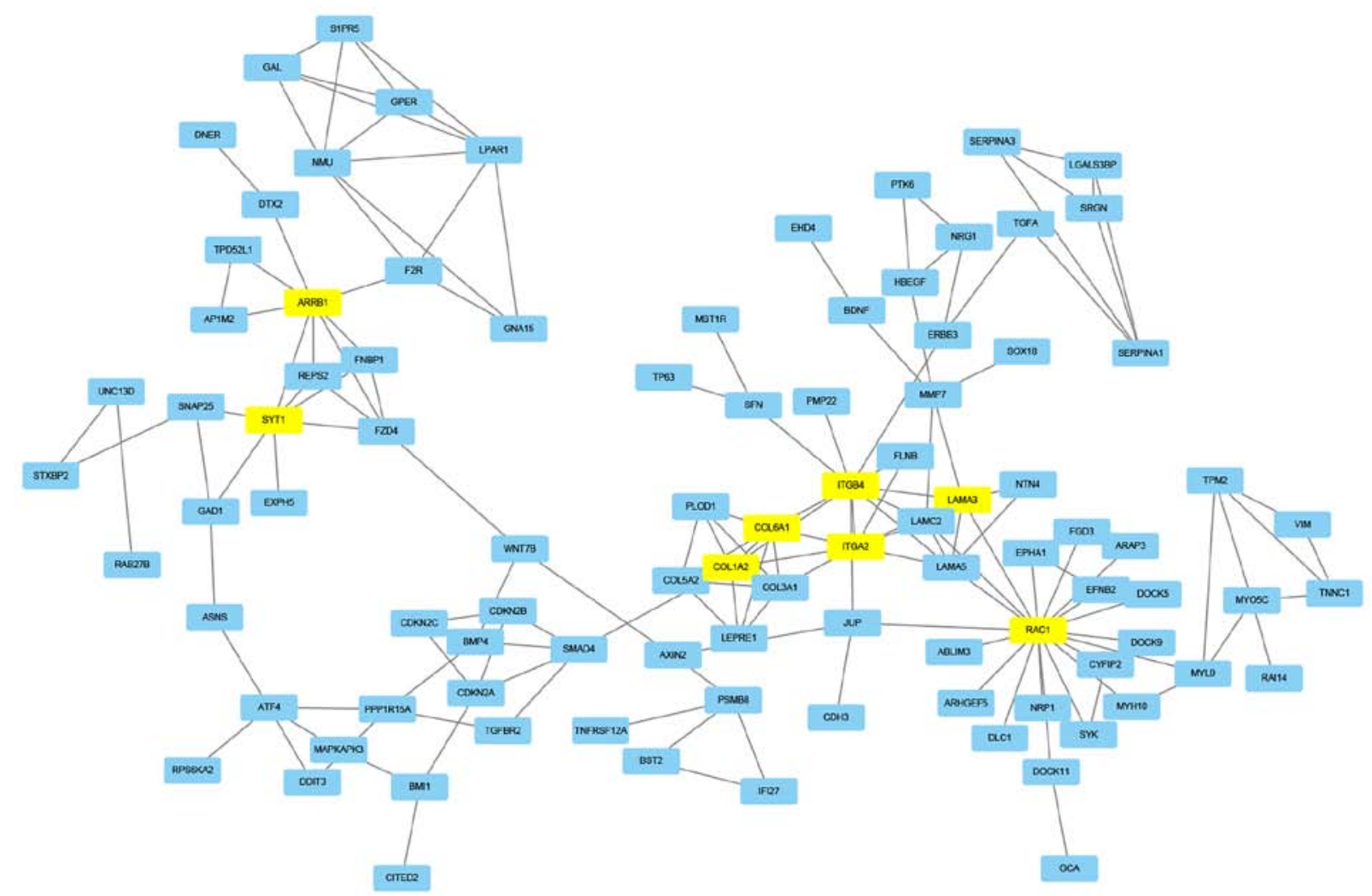

Figure 3. Protein-protein interaction network. The interaction network chart of the eight node proteins containing ARRB1, SYT1, COL1A2, COL6A1, ITGB4, ITGA2, LAMA3 and RAC1, which were associated with other node proteins ( $>7$ proteins). ARBB1, arrestin $\beta 1$; COL1A2, collagen type I $\alpha 2$ chain; COL6A1, collagen type VI $\alpha 1$ chain; ITGA2, integrin subunit $\alpha 2$; ITGB4, integrin subunit $\beta$ 4; LAMA3, laminin subunit $\alpha$ 3; RAC1, Rac family small GTPase 1; SYT1, synaptotagmin 1.

levels of each gene (Breslow $\mathrm{P}=3.40 \times 10^{-2}, 2.40 \times 10^{-2}$, logrank $\mathrm{P}=3.09 \times 10^{-2}, 1.27 \times 10^{-5}$ and $9.7 \times 10^{-3}$, respectively). This suggested that the selected genes may contribute to dasatinib resistance in pancreatic cancer.

\section{Discussion}

In pancreatic cancer, vascular endothelial growth factor receptor (VEGFR), epidermal growth factor receptor (EGFR), insulin-like growth factor 1 receptor (IGF1R) or matrix metalloproteinases (MMPs), such as MMP-9, are commonly overexpressed, which allows treatment of pancreatic cancer using certain small molecule inhibitors against these proteins $(22,23)$. Dasatinib is a potent receptor tyrosine kinase/Src inhibitor, which reduces pancreatic tumor cell growth in vitro (11). Dasatinib is also FDA-approved for the treatment of pancreatic tumors (11). Previous studies have reported that increased activation of Src kinases, which are molecular targets of dasatinib, is associated with poor survival in patients with pancreatic cancer (15). In these patients, co-treatment with dasatinib and gemcitabine may contribute to disease stability (24). However, drug resistance frequently develops rapidly. Dasatinib resistance is a major challenge for the treatment of patients with pancreatic cancer (25). It is therefore crucial to determine the underlying mechanisms of dasatinib resistance in order to develop appropriate therapeutic strategies against drug resistance. In the present study, DEGs were identified between dasatinib-sensitive and dasatinib-resistant pancreatic cancer cells. GO annotation and pathway enrichment were then performed to analyze the functions of theses DEGs. The association between these DEGs was analyzed by constructing a PPI network. In addition, Kaplan-Meier survival analysis revealed that some DEGs were highly associated with the prognosis of patients with pancreatic cancer. Numerous hub genes were confirmed to serve a key role in dasatinib resistance, and may therefore be considered as potent molecular targets for the treatment of dasatinib-resistance in pancreatic cancer.

A total of 472 DEGs between dasatinib-resistant and dasatinib-sensitive pancreatic cancer cells were identified, including 139 upregulated and 333 downregulated DEGs. Amongst these DEGs, some had a significant fold change $(>3)$ in dasatinib-resistant cancer cells compared with dasatinib-sensitive cells. Vimentin (VIM), transmembrane 41 six family member 18 , serglycin, keratin 7 , lipocalin 2 (LCN2), tumor associated calcium signal transducer 2 and S100 calcium binding protein $\mathrm{P}$ had 5.05, 3.89, 3.71, $-4.35,-4.4,-5.04$ and -5.27 fold changes, respectively. Therefore, these DEGs may represent biomarkers of dasatinib resistance. However, the molecular mechanisms underlying dasatinib resistance remain unclear and require further investigation. Previous studies have reported that these DEGs may contribute to pancreatic cancer in different ways. For example, VIM, which is an important factor that coordinates epithelial to mesenchymal transition and is therefore associated with pancreatic tumor aggressiveness (26), was an upregulated DEG in dasatinib-resistant cell lines in the present study. VIM also participates in colonic neoplastic progression and resistance to histone deacetylase inhibitors (27). 

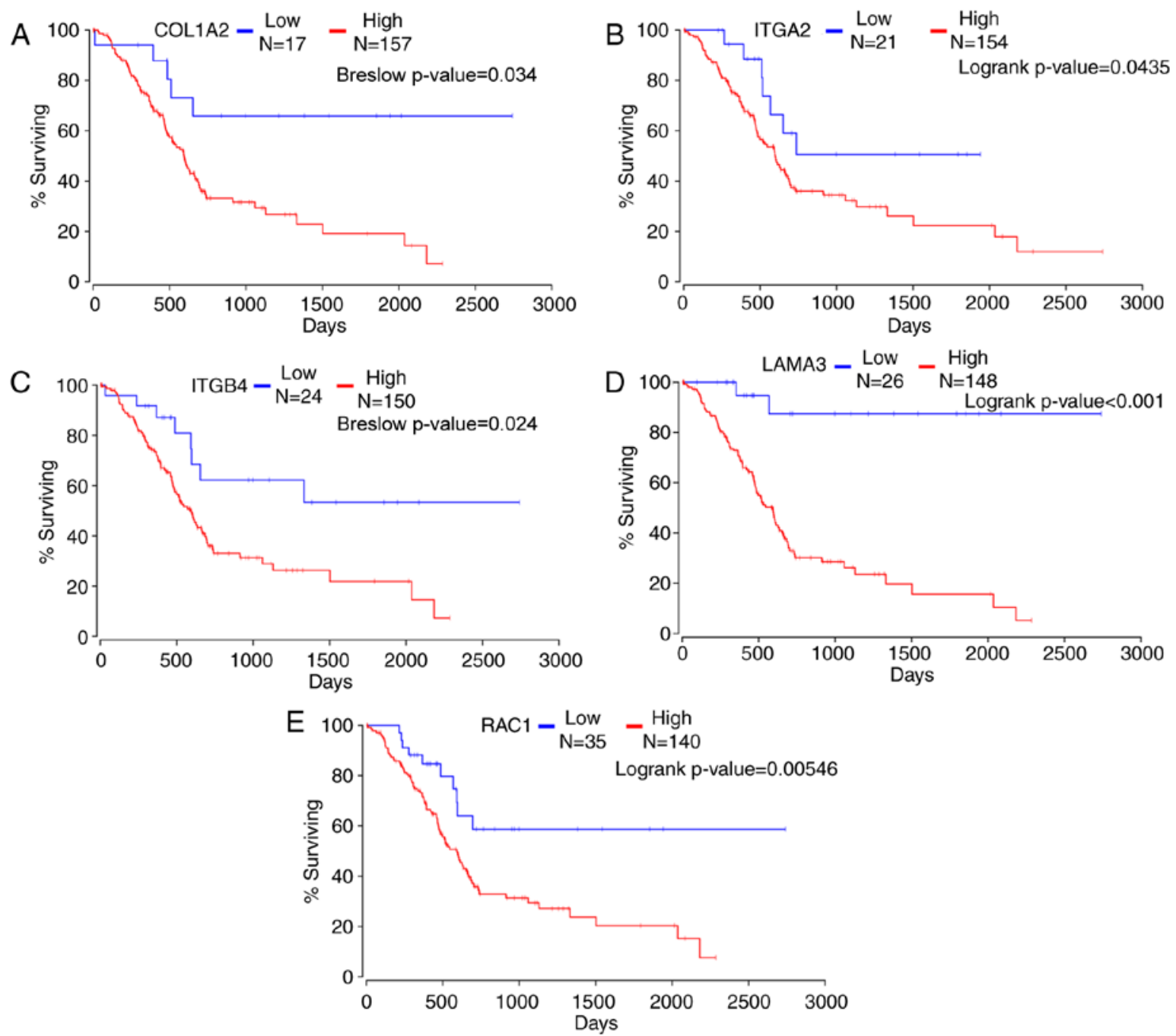

Figure 4. The relationship between hub genes and pancreatic adenocarcinoma prognosis. Kaplan-Meier survival analyses indicated that patients with the higher expression of (A) COL1A2, (B) ITGA2, (C) ITGB4, (D) LAMA3 and (E) RAC1 were associated with poorer survival. COL1A2, collagen type I $\alpha 2$ chain; ITGA2, integrin subunit $\alpha$ 2; ITGB4, integrin subunit $\beta$ 4; LAMA3, laminin subunit $\alpha 3$; RAC1, Rac family small GTPase 1.

Conversely, methylthioadenosine phosphorylase (MATP), which has been considered as a potential therapeutic target for cancer cells with a 9p21 deletion (28), was a downregulated DEG in dasatinib-resistant cell lines in the present study. A homozygous deletion of MATP with p16INK/CDKN2A has often been reported in invasive pancreatic cancer (29). In addition, BCL2 interacting killer (BIK) was a downregulated DEG in dasatinib-resistant cells in a previous study (11). ERK1/2 degrades BIK in SRC-, BRAF- or KRAS-activated tumors (30). In conclusion, the DEGs identified in the present study may influence the progression and development of pancreatic cancer, and may contribute to dasatinib resistance through intricate molecular mechanisms.

The biological functions of the DEGs identified in the present study were determined by GO annotation using the DAVID database. In the CC ontology, the majority of enriched DEGs were associated with cell membrane components, whereas a number of enriched DEGs were associated with non-membrane-related components, including the actin cytoskeleton and extracellular exosome. Mithraprabhu et al (31), reported that the actin cytoskeleton may be involved in the drug resistance of numerous cancers. The results of the
CC ontology in the present study demonstrated that the development of dasatinib resistance was caused by complex cellular mechanisms and may be associated with membrane and non-membrane cellular structure. The results of the BP ontology indicated that the regulation-associated components were the most enriched, including the regulation of signaling pathways, such as 'signal regulation' (118 genes) and 'cell communication regulation' (118 genes). The remaining enriched categories were associated with cell proliferation, transduction, apoptosis and localization, including 'cell proliferation' (95 genes), 'intracellular signal transduction' (100 genes), 'apoptotic processes' (73 genes) and 'macromolecule localization' (97 genes).

Drug resistance is associated with abnormalities in signaling pathways, such as the PI3K/AKT/mTOR signaling pathway (32). A drug is a foreign chemical capable of damaging tumor cells, and drug resistance is considered as a cellular adaption to drugs. Regulation of signaling pathways is therefore a key factor in the development of drug resistance. In response to dasatinib, tumor cells are able to develop resistance through mutation or activation of certain pathways. For instance, the MAPK pathway may contribute to the resistance 
of dasatinib (18). During the adaptation process, numerous gene alterations occur in tumors and may lead to signaling pathway dysfunction, which may influence cell proliferation, transduction, apoptosis and localization (33) and result in persistent cell proliferation, even when tumor cells are exposed to the drug (34). The results of the present study indicated that isolated DEGs may affect certain molecular mechanisms associated with dasatinib resistance in pancreatic tumors. In the MF ontology, most categories comprised of regulation and binding-associated components, including 'molecular function regulation' and 'calcium ion binding'. These results suggested that the highlighted DEGs may participate in the regulation of molecular function and ionic pumps. The 'molecular function regulator' GO term is associated with drug resistance (35), and some DEGs identified in the present study may have significant molecular functions. For example, a previous study reported that VIM is associated with drug resistance (36). In addition, LCN2 enhances migration and cisplatin resistance in endometrial cell carcinoma (37). Ion pumps also confer drug resistance in various types of cancer, such as gastric, colorectal and lung cancer (38). The DEGs highlighted in the present study may therefore serve crucial roles in dasatinib resistance through regulator-associated components and/or binding-associated components. However, further investigation is necessary to confirm these hypotheses.

Pathway analysis may provide additional information on biological gene function compared with GO analysis. In the present study, significant pathways were identified, including the PI3K/Akt and MAPK signaling pathways. Signaling pathways in cancer may participate in the development of drug resistance; for example, Guo et al (39) have reported that the P38/MAPK pathway serves an important role in the chemotherapy resistance of vincristine in gastric cancer cells. In addition, the PI3K/Akt and MAPK signaling pathways are crucial for cancer development, since they are involved in cell proliferation, maintenance, invasion, drug-resistance and metastasis $(40,41)$. However, the association between the molecular mechanism of dasatinib resistance and these signaling pathways remains unknown and requires further investigation.

Through the construction of a PPI network, the results from the present study demonstrated that the local network comprised a series of hub proteins, including ARRB1, SYT1, COL1A2, COL6A1, ITGB4, ITGA2, LAMA3 and RAC1. It has been previously reported that these genes are associated with drug resistance and cancer progression; for example, ARRB1 is an epigenetic regulator and an important molecule in signal transduction pathways (42). Rosanò et al (43) reported that ARRB1 participates in the Wnt signaling pathway, which leads to chemotherapy resistance in ovarian tumor cells. In addition, SYT1 may contribute to cellular resistance to environmental stresses, such as mechanical stress (44). Jun et al (45) revealed that SYT1 increases lung cancer invasiveness through the activation of CD74-ROS, which is a fusion kinase in lung cancer. COL1A 2 and COL6A1 are crucial molecules associated with drug resistance, and increased expression of COL1A2 is involved in drug resistance in ovarian cancer cells (46). In addition, COL6A1 promotes cancer growth and is upregulated in castration-resistant tumors (47). Furthermore, integrins are related to transmembrane glycoprotein receptors in a non-covalent way; ITGB4 is associated with the progression of taxane-resistant prostate cancer (48), and Nones et al (49) reported that ITGA2 hypomethylation is associated with its high expression and with poor survival in PDAC. In the present study, COL1A2, COL6A1, ITGB4, ITGA2 and LAMA3 were associated with the PI3K/AKT signaling pathway, which indicated that the PI3K/AKT signaling pathway was enriched in cancer. RAC1 serves key roles in a number of signaling pathways, including the PI3K/AKT and the MAPK signaling pathways $(50,51)$. RAC1 is therefore a focal point for research on tumor resistance (52). Wang et al (53) reported that RAC1 can promote chemotherapy resistance in leukemia cells through the activation of Rac1 GTPase. Previous studies demonstrated that mutations in RAC1 P29S induce RAF inhibitor resistance in melanoma cells (54). RAC1 was highly associated with drug resistance through different signaling pathways based on the results of the GO and KEGG term analysis in the present study. Furthermore, the association between the aforementioned hub genes and the survival curves for pancreatic adenocarcinoma revealed that five hub genes (COL1A2, ITGB4, ITGA2, LAMA3 and RAC1) were significantly associated with the overall survival of patients with pancreatic cancer. These findings supported the hypothesis that these hub genes may serve crucial roles in dasatinib resistance. However, whether certain distinct molecular mechanisms of dasatinib resistance exist, and whether these genes can be targets for the treatment of dasatinib resistant pancreatic carcinoma remain unclear.

In conclusion, the results from the present study revealed certain distinct mechanisms of dasatinib resistance in pancreatic cancer. Bioinformatics approaches were used to determine the DEGs in dasatinib-resistant pancreatic cancer cells. The aberrant signaling pathways were associated with dasatinib-resistant pancreatic tumors. In addition, five hub genes were identified as potential targets for the treatment of dasatinib resistance. The results from the current study may provide a valuable insight for the determination of dasatinib-resistance in pancreatic cancer. However, further investigation is required.

\section{Acknowledgements}

Not applicable.

\section{Funding}

The present study was supported by The National Natural Science Foundation of China (grant nos. 81572928 and 81772978).

\section{Availability of data and materials}

The datasets generated and/or analyzed during the present study are available in the 'Dasatinib data' repository (https://pan.baidu.com/s/19wCo7dp_Rt2ninY1vGbSMw).

\section{Authors' contributions}

JSW and RBH analyzed the data and wrote the manuscript. XYS, YTC, JFS, XWC, HHZ, YG and XC wrote parts of the manuscript. JFC revised the manuscript. JSW, RBH, XYS, 
YTC, JFS, XWC, HHZ, YG, XC and JFC participated in the statistical analysis and contributed to the interpretation of the results as well as the writing of the manuscript. All authors read and approved the final manuscript.

\section{Ethics approval and consent to participate}

Not applicable.

\section{Patients consent for publication}

Not applicable.

\section{Competing interests}

The authors declare that they have no competing interests.

\section{References}

1. Jemal A, Bray F, Center MM, Ferlay J, Ward E and Forman D: Global cancer statistics. CA Cancer J Clin 61: 69-90, 2011.

2. Siegel R, Naishadham D and Jemal A: Cancer statistics, 2013. CA Cancer J Clin 63: 11-30, 2013.

3. Lau MK, Davila JA and Shaib YH: Incidence and survival of pancreatic head and body and tail cancers: A population-based study in the United States. Pancreas 39: 458-462, 2010.

4. Quaresma M, Coleman MP and Rachet B: 40-year trends in an index of survival for all cancers combined and survival adjusted for age and sex for each cancer in England and Wales, 1971-2011: A population-based study. Lancet 385: 1206-1218, 2015.

5. Reya T, Morrison SJ, Clarke MF and Weissman IL: Stem cells, cancer, and cancer stem cells. Nature 414: 105-111, 2001.

6. Shah NP, Tran C, Lee FY, Chen P, Norris D and Sawyers CL: Overriding imatinib resistance with a novel ABL kinase inhibitor. Science 305: 399-401, 2004.

7. Keating GM: Dasatinib: A review in chronic myeloid leukaemia and Ph+ acute lymphoblastic leukaemia. Drugs 77: 85-96, 2017.

8. Summy JM and Gallick GE: Src family kinases in tumor progression and metastasis. Cancer Metastasis Rev 22: 337-358, 2003.

9. Koon HK, Chan PS, Wong RN, Wu ZG, Lung ML, Chang CK and Mak NK: Targeted inhibition of the EGFR pathways enhances Zn-BC-AM PDT-induced apoptosis in well-differentiated nasopharyngeal carcinoma cells. J Cell Biochem 108: 1356-1363, 2009.

10. Wang X, Wilson MJ, Slaton JW, Sinha AA, Ewing SL and Pei D Increased aggressiveness of human prostate PC-3 tumor cells expressing cell surface localized membrane type-1 matrix metalloproteinase (MT1-MMP). J Androl 30: 259-274, 2009.

11. Chien W, Sun QY, Lee KL, Ding LW, Wuensche P, Torres-Fernandez LA, Tan SZ, Tokatly I, Zaiden N, Poellinger L, et al: Activation of protein phosphatase 2A tumor suppressor as potential treatment of pancreatic cancer. Mol Oncol 9: 889-905, 2015

12. Gnoni A, Marech I, Silvestris N, Vacca A and Lorusso V: Dasatinib: An anti-tumour agent via Src inhibition. Curr Drug Targets 12: 563-578, 2011.

13. Trevino JG, Summy JM, Lesslie DP, Parikh NU, Hong DS Lee FY, Donato NJ, Abbruzzese JL, Baker CH and Gallick GE: Inhibition of SRC expression and activity inhibits tumor progression and metastasis of human pancreatic adenocarcinoma cells in an orthotopic nude mouse model. Am J Pathol 168: 962-972, 2006.

14. Nagaraj NS, Smith JJ, Revetta F, Washington MK and Merchant NB: Targeted inhibition of Src kinase signaling attenuates pancreatic tumorigenesis. Mol Cancer Ther 9: 2322-2332, 2010.

15. Morton JP, Karim SA, Graham K, Timpson P, Jamieson N, Athineos D, Doyle B, McKay C, Heung MY, Oien KA, et al: Dasatinib inhibits the development of metastases in a mouse mode of pancreatic ductal adenocarcinoma. Gastroenterology 139 292-303, 2010

16. Bartscht T, Rosien B, Rades D, Kaufmann R, Biersack H, Lehnert H, Gieseler F and Ungefroren H: Dasatinib blocks transcriptional and promigratory responses to transforming growth factor-beta in pancreatic adenocarcinoma cells through inhibition of Smad signalling: Implications for in vivo mode of action. Mol Cancer 14: 199, 2015.
17. Bartscht T, Rosien B, Rades D, Kaufmann R, Biersack H, Lehnert H, Gieseler F and Ungefroren H: Dasatinib blocks transcriptional and promigratory responses to transforming growth factor-beta in pancreatic adenocarcinoma cells through inhibition of Smad signalling: Implications for in vivo mode of action. Mol Cancer 14: 199, 2015.

18. Beadnell TC, Mishall KM, Zhou Q, Riffert SM, Wuensch KE, Kessler BE, Corpuz ML, Jing X, Kim J, Wang G, et al: The mitogen-activated protein kinase pathway facilitates resistance to the src inhibitor dasatinib in thyroid cancer. Mol Cancer Ther 15: 1952-1963, 2016.

19. Beauchamp EM, Woods BA, Dulak AM, Tan L, Xu C, Gray NS, Bass AJ, Wong KK, Meyerson M and Hammerman PS: Acquired resistance to dasatinib in lung cancer cell lines conferred by DDR2 gatekeeper mutation and NF1 loss. Mol Cancer Ther 13: 475-482, 2014

20. Lilian A, Denis S, Johnston JB and Raquel A: p53 and autophagy contribute to dasatinib resistance in primary CLL lymphocytes. Leuk Res 35: 99-102, 2011.

21. Zhang L, Huang Y, Zhuo W, Zhu Y, Zhu B and Chen Z: Identification and characterization of biomarkers and their functions for Lapatinib-resistant breast cancer. Med Oncol 34: 89, 2017.

22. Andrikou K, Peterle C, Pipitone S, Salati M and Cascinu S: Emerging antibodies for the treatment of pancreatic cancer. Expert Opin Emerg Drugs 22: 39-51, 2017.

23. Knapinska AM, Estrada CA and Fields GB: The roles of matrix metalloproteinases in pancreatic cancer. Prog Mol Biol Transl Sci 148: 339-354, 2017.

24. Hong DS, Choe JH, Naing A, Wheler JJ,Falchook GS, Piha-Paul S, Moulder SL, George GC, Choe JM, Strauss LC, et al: A phase 1 study of gemcitabine combined with dasatinib in patients with advanced solid tumors. Invest New Drugs 31: 918-926, 2013.

25. Chen Z, Zhao L, Shi K and Chen B: Mechanism comparison of gemcitabine and dasatinib-resistant pancreatic cancer by integrating mRNA and miRNA expression profiles. Clin Lab 64: 749-757, 2018

26. Shah AN, Summy JM,Zhang J,Park SI,Parikh NU and Gallick GE: Development and characterization of gemcitabine-resistant pancreatic tumor cells. Ann Surg Oncol 14: 3629, 2007.

27. Lazarova DL and Bordonaro M: Vimentin, colon cancer progression and resistance to butyrate and other HDAC is. J Cell Mol Med 20: 989-993, 2016.

28. Efferth T, Gebhart E, Ross DD and Sauerbrey A: Identification of gene expression profiles predicting tumor cell response to L-alanosine. Biochem Pharmacol 66: 613-621, 2003

29. Hustinx SR, Hruban RH, Leoni LM, Iacobuzio-Donahue C, Cameron JL, Yeo CJ, Brown PN, Argani P, Ashfaq R, Fukushima N, et al: Homozygous deletion of the MTAP gene in invasive adenocarcinoma of the pancreas and in periampullary cancer: A potential new target for therapy. Cancer Biol Ther 4: 83-86, 2005.

30. Lopez J, Hesling C, Prudent J, Popgeorgiev N, Gadet R, Mikaelian I, Rimokh R, Gillet G and Gonzalo P: Src tyrosine kinase inhibits apoptosis through the Erk1l[sol]|2-dependent degradation of the death accelerator Bik. Cell Death Differ 19: 1459-1469, 2012

31. Mithraprabhu S, Khong T and Spencer A: Overcoming inherent resistance to histone deacetylase inhibitors in multiple myeloma cells by targeting pathways integral to the actin cytoskeleton. Cell Death Dis 5: e1134, 2014.

32. Guerrero-Zotano A,MayerIA and ArteagaCL:PI3K/AKT/mTOR: Role in breast cancer progression, drug resistance, and treatment. Cancer Metastasis Rev 35: 515-524, 2016.

33. Guri $\mathrm{Y}$ and Hall MN: mTOR signaling confers resistance to targeted cancer drugs. Trends Cancer 2: 688-697, 2016.

34. Xu Z, Mei J and Tan Y: Baicalin attenuates DDP (cisplatin) resistance in lung cancer by downregulating MARK2 and p-Akt. Int J Oncol 50: 93-100, 2017.

35. Kim MH and Kim J: Role of YAP/TAZ transcriptional regulators in resistance to anti-cancer therapies. Cell Mol Life Sci 74 1457-1474, 2017.

36. Xili Y, Yue L, Jianhua Z,Fei G, Dinghong M, Nianyun W, Guohui L and Guanghua G: Analysis of the mechanism of drug resistance of VIM-2-type metallo- $\beta$-lactamase-producing Acinetobacter baumannii isolated from burn patients and its homology. Zhonghua shao shang za zhi (Chinese) 31: 205-210, 2015.

37. Miyamoto T, Kashima H, Yamada Y, Kobara H, Asaka R, Ando H, Higuchi S, Ida K, Mvunta DH and Shiozawa T: Lipocalin 2 enhances migration and resistance against cisplatin in endometrial carcinoma cells. PLoS One 11: e0155220, 2016. 
38. Eljack ND, Ma HY, Drucker J, Shen C, Hambley TW, New EJ, Friedrich T and Clarke RJ: Mechanisms of cell uptake and toxicity of the anticancer drug cisplatin. Metallomics 6: 2126-2133, 2014

39. Guo X, Ma N, Wang J, Song J, Bu X, Cheng Y, Sun K, Xiong H, Jiang G, Zhang B, et al: Increased p38-MAPK is responsible for chemotherapy resistance in human gastric cancer cells. BMC Cancer 8: 375, 2008.

40. Ersahin T, Tuncbag N and Cetin-Atalay R: The PI3K/AKT/mTOR interactive pathway. Mol Biosyst 11: 1946-1954, 2015.

41. Xinling Z, Leina M, Jieqiong Q, Hui S, Wengong Y and Yuchao G: MAPK/ERK signaling pathway-induced hyper-O-GlcNAcylation enhances cancer malignancy. Mol Cell Biochem 410: 101-110, 2015.

42. Shenoy SK and Lefkowitz RJ: $\beta$-Arrestin-mediated receptor trafficking and signal transduction. Trends Pharmacol Sci 32: 521-533, 2011.

43. Rosanò L, Cianfrocca R, Tocci P, Spinella F, Di Castro V, Caprara V, Semprucci E, Ferrandina G, Natali PG and Bagnato A: Endothelin A receptor/ $\beta$-arrestin signaling to the Wnt pathway renders ovarian cancer cells resistant to chemotherapy. Cancer Res 74: 7453-7464, 2014.

44. Pérez-Sancho J, Vanneste S, Lee E, McFarlane HE, Esteban Del Valle A, Valpuesta V, Friml J, Botella MA and Rosado A: The Arabidopsis synaptotagmin1 is enriched in endoplasmic reticulum-plasma membrane contact sites and confers cellular resistance to mechanical stresses. Plant Physiol 168 : 132-143, 2015.

45. Jun HJ, Johnson H, Bronson RT, de Feraudy S, White F and Charest A: The oncogenic lung cancer fusion kinase CD74-ROS activates a novel invasiveness pathway through E-Syt1 phosphorylation. Cancer Res 72: 3764-3774, 2012.

46. Januchowski R, Świerczewska M, Sterzyńska K, Wojtowicz K, Nowicki $M$ and Zabel $M$ : Increased expression of several collagen genes is associated with drug resistance in ovarian cancer cell lines. J Cancer 7: 1295-1310, 2016.

47. Zhu YP, Wan FN, Shen YJ, Wang HK, Zhang GM and Ye DW: Reactive stroma component COL6A1 is upregulated in castration-resistant prostate cancer and promotes tumor growth. Oncotarget 6: 14488-14496, 2015.
48. Kawakami K, Fujita Y, Kato T, Mizutani K, Kameyama K, Tsumoto H, Miura Y, Deguchi T and Ito M: Integrin $\beta 4$ and vinculin contained in exosomes are potential markers for progression of prostate cancer associated with taxane-resistance. Int J Oncol 47: 384-390, 2015.

49. Nones K, Waddell N, Song S, Patch AM, Miller D, Johns A, Wu J, Kassahn KS, Wood D, Bailey P, et al: Genome-wide DNA methylation patterns in pancreatic ductal adenocarcinoma reveal epigenetic deregulation of SLIT-ROBO, ITGA2 and MET signaling. Int J Cancer 135: 1110-1118, 2014.

50. Zhou G, Peng F, Zhong Y, Chen Y, Tang M and Li D: Rhein suppresses matrix metalloproteinase production by regulating the Rac1/ROS/MAPK/AP-1 pathway in human ovarian carcinoma cells. Int J Oncol 50: 933-941, 2017.

51. Pan Y, Wang N, Xia P, Wang E, Guo Q and Ye Z: Inhibition of Racl ameliorates neuronal oxidative stress damage via reducing Bcl-2/Rac1 complex formation in mitochondria through PI3K/Akt/mTOR pathway. Exp Neurol 300: 149-166, 2018.

52. Zhang $\mathrm{T}$ and Wang $\mathrm{N}$ : miR-135a confers resistance to gefitinib in non-small cell lung cancer cells by upregulation of RAC1. Oncol Res 26: 1191-1200, 2018.

53. Wang JY, Yu P, Chen S, Xing H, Chen Y, Wang M, Tang K, Tian Z, Rao Q and Wang J: Activation of Racl GTPase promotes leukemia cell chemotherapy resistance, quiescence and niche interaction. Mol Oncol 7: 907-916, 2013.

54. Watson IR, Li L, Cabeceiras PK, Mahdavi M, Gutschner T, Genovese G, Wang G, Fang Z, Tepper JM, Stemke-Hale K, et al: The RAC1 P29S hotspot mutation in melanoma confers resistance to Pharmacological inhibition of RAF. Cancer Res 74: 4845,2014 